\title{
Development and Validation of Network Clock Measurement Techniques
}

\author{
Karen F. O'Donoghue and Timothy R. Plunkett \\ System Research and Technology Department \\ Combat Systems Branch \\ Naval Surface Warfare Center, Dahlgren Division \\ Dahlgren, Virginia 22448-5000
}

\begin{abstract}
Navy systems are inherently real-time. As these systems evolve with the introduction of new requirements, technologies, and commercial-off-theshelf (COTS) equipment, they are evolving toward more distributed systems. A key component to the deployment of real-time distributed systems in Navy applications is quality clock synchronization among the various computing platforms in the distributed system. The Network Time Protocol (NTP) is being viewed as a key technology for the provision of this synchronization. To facilitate analysis of NTP, a software mechanism for the measurement of clock offsets has been developed. This mechanism has been validated using independent validation tests. Finally, this mechanism can be used to study NTP and time synchronization subnet behavior.
\end{abstract}

Keywords: Time, Clock, Synchronization, NTP

\section{1: Introduction}

Navy platforms are composed of a number of systems, each with several components, that carry out functions ranging from basic ship operation and navigation to control of sophisticated sensor and weapon systems. All of these systems require a common understanding of time to perform their inter-related functions. These systems have traditionally been implemented with Navy unique computers interconnected via Navy unique interfaces. In addition, the time synchronization systems have traditionally been implemented using separate time distribution networks.

Navy platforms are rapidly evolving. This evolution is being driven by additional application requirements, advancing computer and communication technologies, and the incorporation of Commercial Off The Shelf (COTS) components. These changes are forcing the
Navy to reconsider its fundamental approaches to deploying systems.

A key area impacted by the evolution of Navy platforms is system wide time synchronization. New approaches for time synchronization have a number of basic goals: (1) The time synchronization solution must meet the accuracy, stability, and reliability requirements of Navy systems; (2) The solution must be capable of being implemented using COTS components and an open standards based communication infrastructure; (3) The solution needs to scale to meet the needs of rapidly evolving and expanding Navy platforms.

The Navy is considering the use of the Network Time Protocol (NTP) [1] in a variety of systems. NTP would provide a basic component for meeting the goals outlined above. NTP is a protocol for the exchange of time information between clocks in computers interconnected by a network based on the Internet Protocol (IP). NTP also includes algorithms that detail how that information is to be used to synchronize these clocks.

A tool was required to evaluate the performance of NTP in Navy systems based on COTS equipment. This tool needed to provide an independent mechanism to dynamically measure clock offset. To meet this requirement, a software based tool was developed. Tests were conducted to validate the results provided by this tool.

\section{1: Basic Definitions}

The following definitions are provided for terms used in this paper. A clock is a device that provides time information to consumers. A clock has two attributes, phase and frequency. Phase is the current value of time for the clock, and frequency is the rate at which the clock increments. Offset is the difference in the phase between two clocks. A synchronization subnet is a collection of computer clocks that exchange time information for the purposes of synchronization. 


\section{2: A Clock Offset Measurement Tool}

The requirement for a Clock Offset Measurement Tool (COMT) has been identified. Below, the objectives, theoretical basis and model, and assumptions are discussed.

\section{1: Tool Objectives}

There were a number of objectives identified for a clock offset measurement tool. First, the tool must provide an accurate measure of the current phase difference between any two clocks utilizing the tool. Next, the tool must be flexible, operating on a multitude of computer platforms connected by a variety of IP based networks. In addition, the tool cannot significantly impact the resource being measured, namely the clock. The tool must be scalable, allowing for the simultaneous measurement of numerous clock pairs without excessive cost. Finally, the tool must be independent of the clock resource and synchronization processes in order to provide a valid analysis tool.

\section{2: Theoretical Basis and Model}

The initial tool design decision, based on the above objectives, was that a clock offset measurement tool based on software mechanisms would be preferable. A mechanism requiring additional hardware would not meet the flexibility or scalability requirements. A software offset measurement mechanism by necessity is going to be a statistical measurement. To provide the foundation for the COMT, the mechanism used by NTP to determine clock offsets and roundtrip delays was chosen.

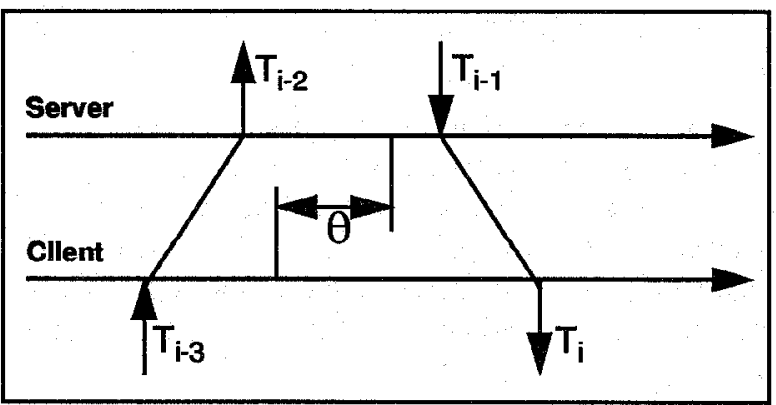

Figure 1. Time Information Exchange

The mechanism chosen for the COTM is based on a returnable time concept that calculates both the offset and the delay with a single exchange of time information. Figure 1 shows the exchange of information between the client and server to obtain four timestamps. These timestamps are then used in the two equations (EQ1 and EQ2) to calculate roundtrip delay ( $\delta$ ) and clock offset $(\theta)$ respectively. [1]

$$
\begin{gathered}
\delta_{i}=\left(T_{i}-T_{i-3}\right)-\left(T_{i-1}-T_{i-2}\right) \\
\theta_{i}=\left(\left(T_{i-2}-T_{i-3}\right)+\left(T_{i-1}-T_{i}\right)\right) / 2
\end{gathered}
$$

The clock offset measurement tool takes a number of successive readings to obtain several sets of $(\theta, \delta)$ pairs. The best estimate of clock offset $(\theta)$ is determined by choosing the offset value associated with the smallest value of roundtrip delay ( $\delta$ ). Based on these objectives and model, a simple software based clock offset measurement tool was implemented. Figure 2 illustrates the basic structure of the tool. Figure 3 provides some sample data obtained using this tool.

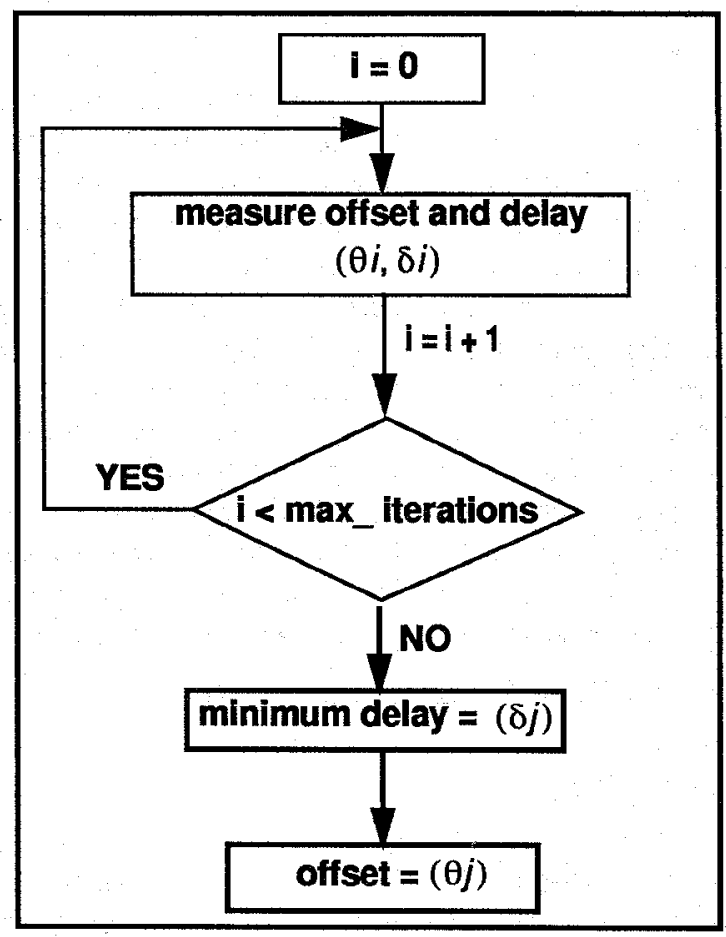

Figure 2. Clock Offset Measurement Tool

\section{3: Assumptions Regarding Offset Calculations}

The approach discussed here includes a number of assumptions that warrant further discussion. There are two assumptions regarding the calculation of clock offset from the exchange of timestamps. One underlying assumption in this calculation is that the two paths that comprise the total roundtrip are symmetric. Thus, it is assumed that the time required to send time information from the client to the server is the same as the time required to send time information from the server to the client. While this may not actually be the case, it is a good estimator for the types of networks anticipated for Navy platforms. Navy platforms are envisioned to consist primarily of relatively 
localized high-speed networks such as Fiber Distributed Data Interface (FDDI) and Asychronous Transfer Mode (ATM). As a result, the difference in network delay encountered on the various paths is negligible compared to the potential for delay in the network interface itself and in the operating system.

A second assumption in the offset calculation is the choice of offset value based on the smallest delay value. The rationale here is that the smallest value of delay between any two clocks represents the least amount of nondeterministic delay in the transaction and is thus the best estimator of the actual offset. This has been demonstrated by data collected in the Internet. [2]

\section{4: Assumptions Regarding Clock Behavior}

This set of assumptions applies to the behavior of the individual clocks involved in the measurement. First, it is assumed that the client and server clocks do not drift apart during the measurement phase. This means the change in offset during the measurement phase is negligible because multiple measurements are taken in quick succession. With this approach, the change in clock offset between the measurements is considered negligible. This enables the tool to factor out any frequency offset or drift components.

The tool also assumes relatively well behaved clocks. This assumption enables the tester to examine the results for a basic sanity check. If two clocks are both monotonically increasing than the offset between the two clocks will be a relatively smooth function. If a single offset value is drastically different from a set of neighboring measurements, measurement error is suspected. The causes of these types of error is a subject for further study.

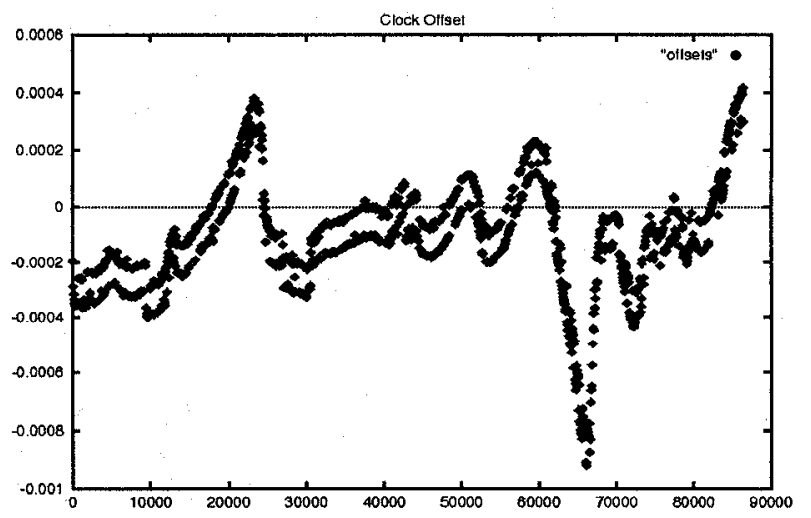

Figure 3. Sample COMT Results

\section{3: Clock Offset Measurement Tool Validation}

NSWCDD developed and implemented a Clock Offset Measurement Tool as described above. As a result, a means was needed to validate this offset measurement tool. Because the above tool is a software based measurement of actual clock offset, a validation test using hardware mechanisms was required. A specialized piece of hardware was available to assist in these measurements. A series of tests were conducted with each test evolving out of the experience gained from the previous tests. This series of tests concluded with the Distributed Time Measurement (DTM) test. The end result was a validation that the measurement tool described above was providing meaningful information concerning clock offsets.

\section{1: Hardware Test Equipment}

The Data Acquisition and Reduction Processor (DARP) is a measurement device developed by NSWCDD and used in the validation tests. The DARP is a system of hardware components which function similarly to a set of logic or performance analyzers. Each of the eight DARP data collections units are individually controlled by specialized hardware and software. Each data collection unit is capable of receiving and timestamping data words up to 32 bits long. The timestamps are received from counters within each of the data collection units. These counters increment based upon a $10 \mathrm{MHz}$ oscillator input. The accuracy of the counters is guaranteed to be within $+/-$ 10 microseconds per second.

\section{2: Initial Validation Testing}

Initial validation testing involved closely coupled systems where either: (1) a single device observed two separate but supposedly simultaneous events or (2) a single event was monitored by independent observers. A number of lessons were learned in this initial validation testing that led to the definition of the actual validation test described below. First, it was difficult to obtain the level of operating system control necessary to precisely generate and detect the events. Without delving into kernel code or monopolizing the resource being observed, it was not possible to precisely generate an event. In addition, standard commercial operating systems do not provide usable access to interrupt scheduling to allow for a nonintrusive detection of an event. Test results improved by monopolizing resources and utilizing real-time mode for the test processes. This, however, was not a desirable approach because it did not accurately model the environment in which the Clock Offset Measurement Tool would be operating. 


\section{3: Distributed Time Measurement Test}

The test ultimately defined for validation purposes was the Distributed Time Measurement (DTM) test. In this test, two computers independently generate and timestamp events (pulses) that are observed by a measurement device, the DARP. The DARP detects, timestamps, and records the occurrence of events generated by both platforms. The difference in the timestamps generated by the DARP represents the amount of time elapsed between the reception of the two pulses. The difference in the timestamps recorded by the computers reflects the time difference in the sending of the two pulses plus the offset between the system clocks of the two computers. By subtracting these two differences, the clock offset can be found. Figure 4 illustrates the basic process. Figure 5 shows the experimental configuration.

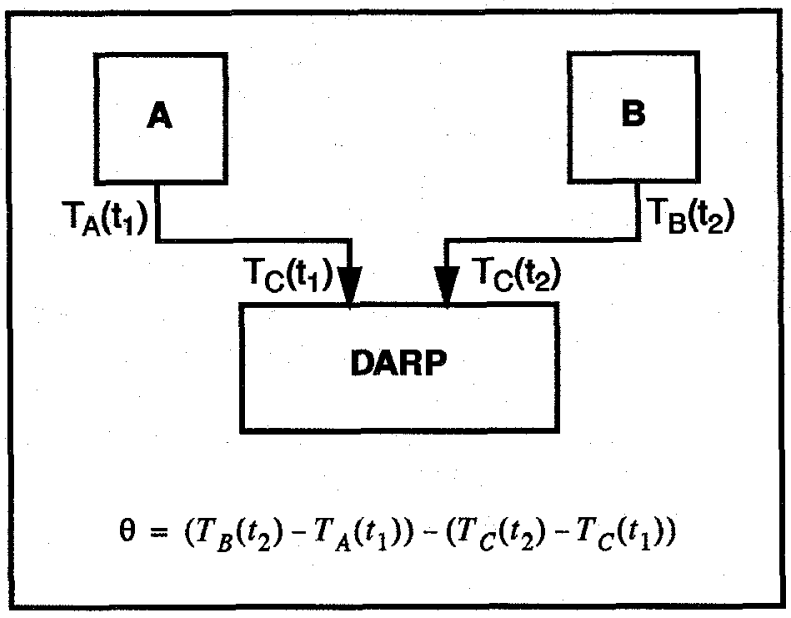

Figure 4. DTM Test Process

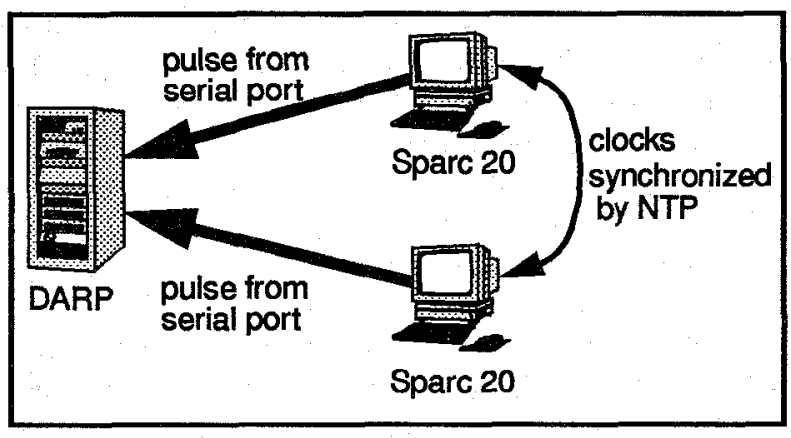

Figure 5. DTM Test Configuration

The clock offset data obtained using this test method was compared with data obtained using the software based Clock Offset Measurement Tool. The difference between the offsets found by the DTM method and the COMT are shown in Figure 6. The results show that a majority of the time the COMT test results agreed with the results of the DTM method to within 0.2 milliseconds. This validation to 0.2 milliseconds is considered acceptable for current Navy testing. It is expected that tools validated to more demanding levels of synchronization will be needed in the future. A review of the offset values recorded by each test seems to indicate that the largest ( 0.5 to 0.8 milliseconds.) differences were due to measurement errors in the DTM. This is based on the assumption that the clocks are well behaved.

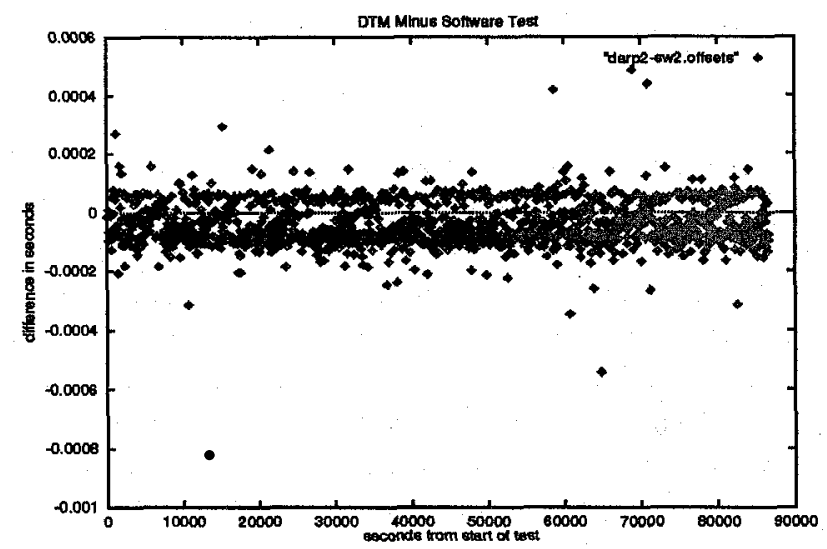

Figure 6. Comparison of DTM and COMT Results

\section{4: Conclusions}

This paper presents the theoretical basis for a tool for measuring computer clock offsets in a COTS based network environment. It discusses steps taken to validate the measurement technique developed. The conclusion reached is that the mechanism for measuring clock offsets is reliable within the stated assumptions. Additionally, it was observed that the sources of difficulty in earlier versions of validation testing point to topics that require further study as Navy systems continue to evolve.

\section{5: References}

[1] David L. Mills, "Network Time Protocol (version 3) Specification, Implementation and Analysis," RFC-1305, March 1992.

[2] David L. Mills, "Internet Time Synchronization: The Network Time Protocol." IEEE Transactions on Communications, Vol. 39, No. 10, pp. 1482-1493, October 1991. 